\title{
Noise sources in the LTP heterodyne interferometer
}

\author{
V Wand ${ }^{1}$, J Bogenstahl ${ }^{2}$, C Braxmaier ${ }^{3,5}$, K Danzmann ${ }^{1}$, A García ${ }^{1}$, \\ F Guzmán ${ }^{1}$, G Heinzel ${ }^{1}$, J Hough ${ }^{2}$, O Jennrich ${ }^{4}$, C Killow ${ }^{2}$, \\ D Robertson ${ }^{2}$, Z Sodnik ${ }^{4}$, F Steier ${ }^{1}$ and H Ward ${ }^{2}$ \\ ${ }^{1}$ Max-Planck-Institut für Gravitationsphysik (Albert-Einstein-Institut) and University of \\ Hannover, Callinstraße 38, D-30167 Hannover, Germany \\ 2 University of Glasgow, UK \\ ${ }^{3}$ EADS Astrium GmbH, Immenstaad, Germany \\ ${ }^{4}$ ESTEC, Noordwijk, The Netherlands \\ E-mail: vinzenz.wand@aei.mpg.de
}

Received 19 October 2005, in final form 23 December 2005

Published 28 March 2006

Online at stacks.iop.org/CQG/23/S159

\begin{abstract}
The LISA Technology Package uses a heterodyne Mach-Zehnder interferometer to monitor the relative motion of the test masses with picometer accuracy. This paper discusses two classes of noise sources that were identified and investigated during the prototype experiments. Most troublesome are electrically induced sidebands on the light, which give rise to nonlinearities in the interferometer output. Even worse, if the differential pathlength between two optical fibres fluctuates, a noise term of milliradian amplitude appears and completely spoils the performance. We discuss the origin and mitigation of this process. Dissimilar beam shapes of the interfering beams produce another type of noise in conjunction with beam jitter and spatially inhomogeneous photodetectors. To study and minimize this effect, we have built a real-time high-resolution phasefront imaging system that will be used for the production of the flight model.
\end{abstract}

PACS numbers: $06.30 . \mathrm{Bp}, 06.30 . \mathrm{Gv}$, 07.60.Ly, 07.87.+v, 42.30.Rx, 95.55.Ym

(Some figures in this article are in colour only in the electronic version)

\section{Introduction}

LISA Pathfinder (LPF) is an ESA technology demonstration mission with contributions from NASA, which is planned for launch in 2009. The main goal of the mission is to verify the performance of the drag-free system controlling two free-floating test masses. In order to monitor these test masses independently from the capacitive sensing system with higher

5 Present address: University of Applied Sciences, Konstanz, Germany. 


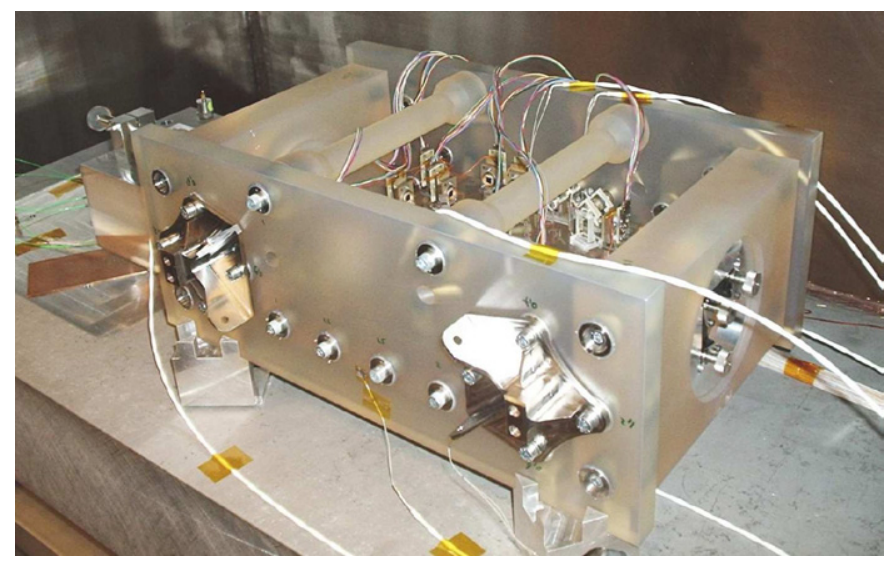

Figure 1. Engineering model of the optical bench. This picture shows the mounting structure (H-bench) made of Zerodur with the optical bench inside. For ground testing the test masses were substituted by gold coated mirrors.

accuracy $\left(10 \mathrm{pm} \mathrm{Hz}^{-\frac{1}{2}}\right)$ and a large dynamic range (many wavelengths), an optical metrology system (OMS) consisting of a set of heterodyne interferometers will be used in this mission $[1,2]$. The baseline concept is a heterodyne Mach-Zender interferometer (MZI) with a heterodyne frequency $f_{\text {het }}$ of $\approx 1 \mathrm{kHz}-2 \mathrm{kHz}$. The optical measuring instrument is divided into two parts:

- The modulation bench consists of a Nd:YAG NPRO laser $(\lambda=1064 \mathrm{~nm})$, a $50 / 50$ beamsplitter and two acousto-optic modulators (both driven by RF signals near $80 \mathrm{MHz}$ ) to shift the frequencies of the two beams such that their frequency difference is $f_{\text {het }}$. Both beams are coupled into polarization-maintaining single-mode optical fibres that also act as mode cleaners. These two fibres route the beams to the optical bench where fibre launchers inject the light into the ultra-stable part of the interferometers.

- The optical bench (see figure 1) contains four non-polarizing interferometers arranged in a Mach-Zehnder topology which are bonded [3] onto a Zerodur baseplate forming a quasi-monolithic unit with high thermal and mechanical stability.

\section{Measuring principle}

The laser beam is split into two parts which are separately frequency shifted by acoustooptic modulators and are brought to interference at a beamcombiner after travelling different paths (figure 2 ). The resulting beat note oscillates with the heterodyne frequency $f_{\text {het }}=$ $10 \mathrm{MHz} / 6160 \approx 1.6233766 \mathrm{kHz}$ generated from a master oscillator running at $10 \mathrm{MHz}$ $[1,2]$.

The heterodyne signal measured at a photodetector is given by

$$
I(t)=A \cdot(1-c \cos (\varphi(t))),
$$

where $I(t)$ is the measured intensity, $A$ is the amplitude of the heterodyne signal and $c$ the interferometric contrast given by

$$
c=\frac{I_{\max }-I_{\min }}{I_{\max }+I_{\min }} .
$$




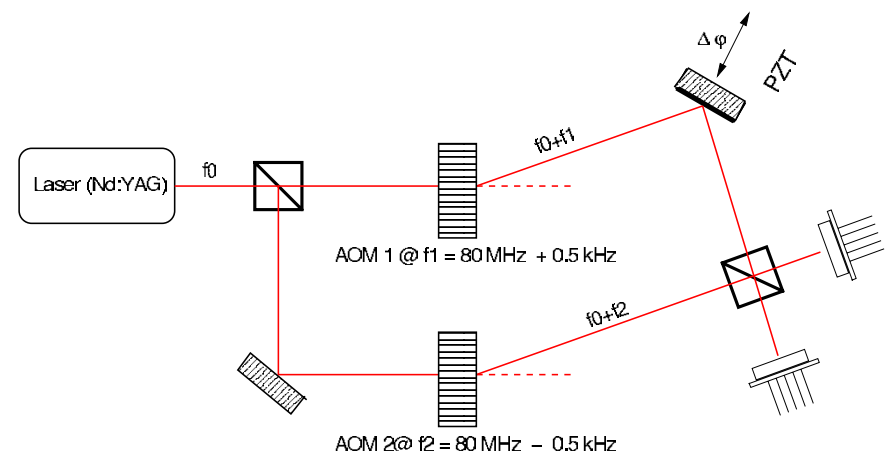

Figure 2. Basic set-up of a non-polarizing heterodyne Mach-Zehnder interferometer. A Nd:YAG laser beam is split into two beams which are separately frequency shifted by an AOM. After travelling different paths the beams are brought to interference. Pathlength variation induced by a piezo actuator (PZT) affects the phase of the interference signal just like test mass motion on LTP.

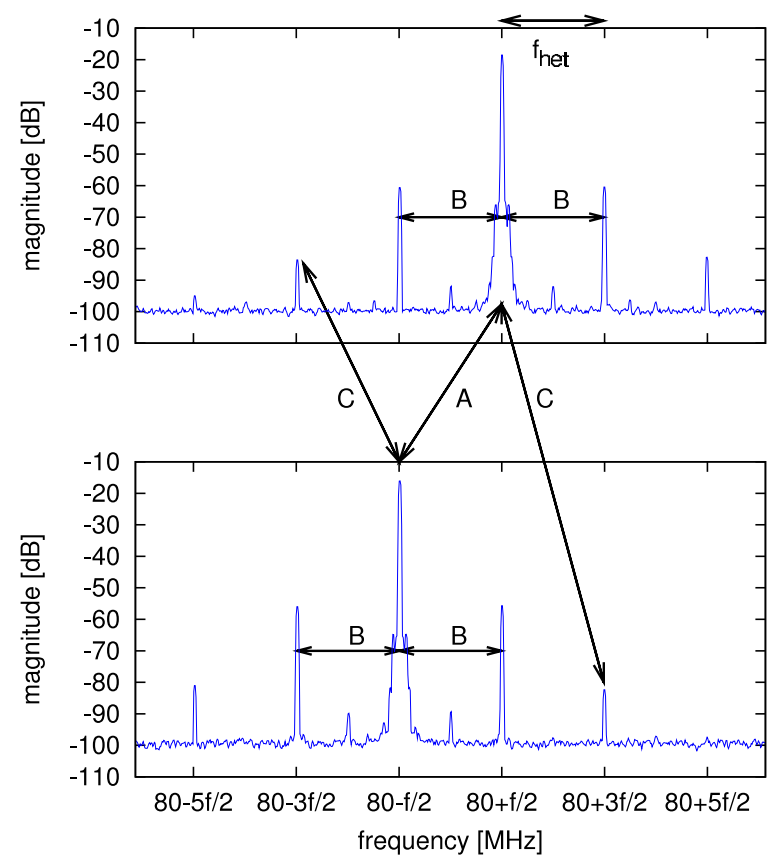

Figure 3. Spectrum of the two RF signals that drive the AOMs show spurious sidebands spaced by multiples of the heterodyne frequency from the carrier. The arrow A indicates the main beat note signal whereas the arrows $\mathrm{B}$ and $\mathrm{C}$ indicate the spurious beat notes.

The phase $\varphi$ of the signal depends on the optical pathlength difference $\Delta L$ between the two arms of the Mach-Zehnder interferometer and can be written as

$$
\varphi(t)=\Delta \omega t+\Delta \varphi=2 \pi f_{\mathrm{het}} t+\frac{2 \pi \Delta L}{\lambda} .
$$

The heterodyne signals are detected by a set of quadrant photodiodes (QPD) that deliver information about the longitudinal pathlength fluctuations and about the alignment of the interferometer (and consequently the test masses) as differential wavefront sensing signals. In a dedicated phasemeter each quadrant is sampled by an ADC at $800 \mathrm{kHz}$ with a resolution of 


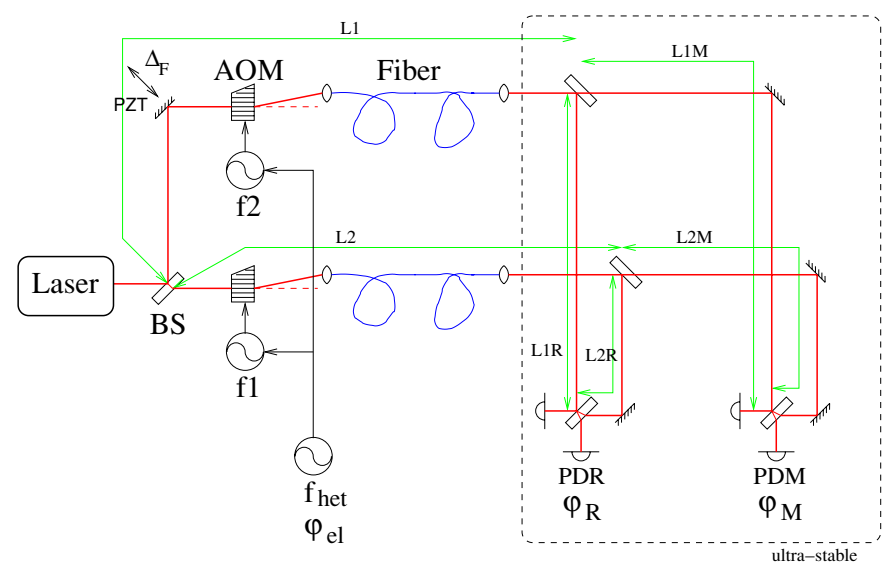

Figure 4. Set-up for the phase error investigation. The diagram shows the ultra-stable part of the interferometer and the unstable part with the modulation bench. The phase difference $\varphi_{\mathrm{M}}-\varphi_{\mathrm{R}}$ is the main measurement which is affected by the occurring phase error.

18 bit. A modified DFT algorithm—single bin discrete Fourier transform (SBDFT) processes the data and reduces the data rate by three orders of magnitude. The complex amplitudes at the heterodyne frequency and the DC component are further processed by a data management unit (DMU) to obtain, among other signals, the output phase of each interferometer [1,2]. To monitor both test masses aboard LTP, four of these MZI are necessary. A first interferometer measures a reference phase $\varphi_{\mathrm{R}}$ which contains all environmental induced pathlength noise such as thermal and mechanical fluctuations that affect the unstable part of the interferometer. This unstable part is the same for all four interferometers (schematically shown in figure 4). Noise occurring in the unstable part affects all interferometers and cancels in the phase differences so that only pathlength variations in the arms of the measurement interferometers will be measured. The two main interferometers measure the relative pathlength fluctuations between the first test mass and the optical bench, and the relative distance of the two test masses to each other. A fourth interferometer is sensitive to laser frequency noise and its output signal is used for active stabilization of the laser. For each of the main longitudinal phase measurements the differential phase between the reference and the relevant measurement interferometer $\varphi=\varphi_{\mathrm{M}}-\varphi_{\mathrm{R}}$ is calculated to determine the actual motion of the test masses.

\section{Residual phase noise at picometer level}

During first phase noise reference measurements, the required sensitivity was not reached within the measurement bandwidth, due to residual phase noise at the milliradian level. The amplitude of this error term depends on the phase difference, $\varphi_{R}-\varphi_{M}$. Figure 4 shows schematically the optical paths in two interferometers. The phases $\varphi_{\mathrm{R}}$ and $\varphi_{\mathrm{M}}$ are defined using the optical pathlengths as follows:

$$
\begin{aligned}
& \varphi_{\mathrm{R}}=\frac{2 \pi}{\lambda}\left(L_{1}+L_{1 \mathrm{R}}-L_{2}-L_{2 \mathrm{R}}\right) \\
& \varphi_{\mathrm{M}}=\frac{2 \pi}{\lambda}\left(L_{1}+L_{1 \mathrm{M}}-L_{2}-L_{2 \mathrm{M}}\right) .
\end{aligned}
$$



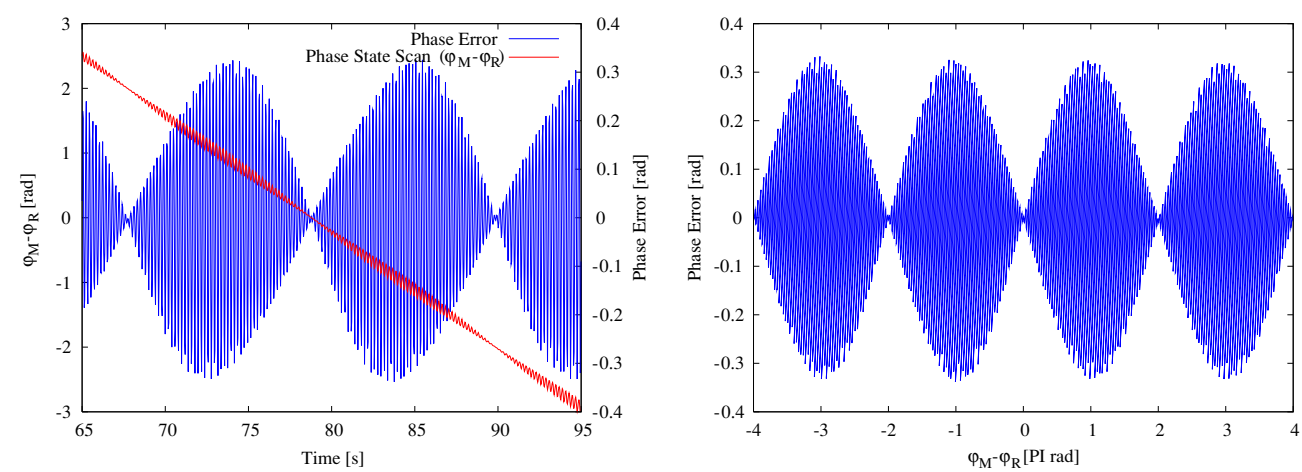

Figure 5. Phase $\varphi=\varphi_{\mathrm{M}}-\varphi_{\mathrm{R}}$ and its error (linear drift substracted) when a strong AM at an offset $f_{\text {het }}$ is added to the RF signal. This illustrates and confirms the $\sin \left(\frac{\varphi_{M}-\varphi_{R}}{2}\right)$ term in equation 8 .

They are measured in the photocurrents from photodiodes PDR and PDM, respectively. Each primary phase measurement is referenced to an electrical master clock that is also used to generate $f_{\text {het }}$. The phase of this electrical reference signal is denoted as $\varphi_{\mathrm{el}}$ (figure 4). Differential pathlength variation on the unstable part of the optical bench that affects both interferometers can be expressed as

$$
\Delta_{\mathrm{F}}=\frac{2 \pi}{\lambda}\left(L_{1}-L_{2}\right)
$$

For the ultra-stable part of the interferometer, the phases

$$
\Delta_{\mathrm{R}}=\frac{2 \pi}{\lambda}\left(L_{1 \mathrm{R}}-L_{2 \mathrm{R}}\right) \quad \text { and } \quad \Delta_{\mathrm{M}}=\frac{2 \pi}{\lambda}\left(L_{1 \mathrm{M}}-L_{2 \mathrm{M}}\right)
$$

represent the differential path length of each individual interferometer. Common mode pathlength fluctuations $\Delta_{\mathrm{F}}$ should cancel completely in the main measurement of $\varphi=\varphi_{\mathrm{R}}-\varphi_{\mathrm{M}}$. This noise cancellation is as expected to the nanometer level, but below that, residual noise remains. Investigations of the spectrum of the AOM driving power showed that the $80 \mathrm{MHz}$ RF signal has spurious sidebands spaced by multiples of the heterodyne frequency from the carrier (figure 3). These sidebands were found to be responsible for the dominant noise source in the interferometer. According to a theoretical analysis, the phase error $\delta \varphi$ caused by first-order sidebands can be described as

$$
\delta \varphi=\left(a \cos \left(\frac{\varphi_{\mathrm{M}}+\varphi_{\mathrm{R}}}{2}\right)+b \sin \left(\frac{\varphi_{\mathrm{M}}+\varphi_{\mathrm{R}}}{2}\right)\right) \cdot \sin \left(\frac{\varphi_{\mathrm{M}}-\varphi_{\mathrm{R}}}{2}\right) .
$$

The phase error caused by second-order sidebands can be described as

$$
\delta \varphi=\left(c \cos \left(\varphi_{\mathrm{M}}+\varphi_{\mathrm{R}}\right)+d \sin \left(\varphi_{\mathrm{M}}+\varphi_{\mathrm{R}}\right)\right) \cdot \sin \left(\varphi_{\mathrm{M}}-\varphi_{\mathrm{R}}\right),
$$

where $a, b, c$ and $d$ are combinations of the amplitudes and phases of the sidebands on the light [2]. In order to verify these formulae, a set of measurements has been taken where additional single sidebands (SSB) and AM at the heterodyne frequency and at twice the heterodyne frequency were added to the RF carrier while the optical path difference was scanned (figures 5 and 6).

These measurements validate the theoretical description of the phase error $\delta \varphi$. A detailed investigation of the origin of the sidebands was conducted. To this end, the purity of the $80 \mathrm{MHz}$ RF signal was measured at the input and outputs of the power amplifier. These measurements indicated the following. 

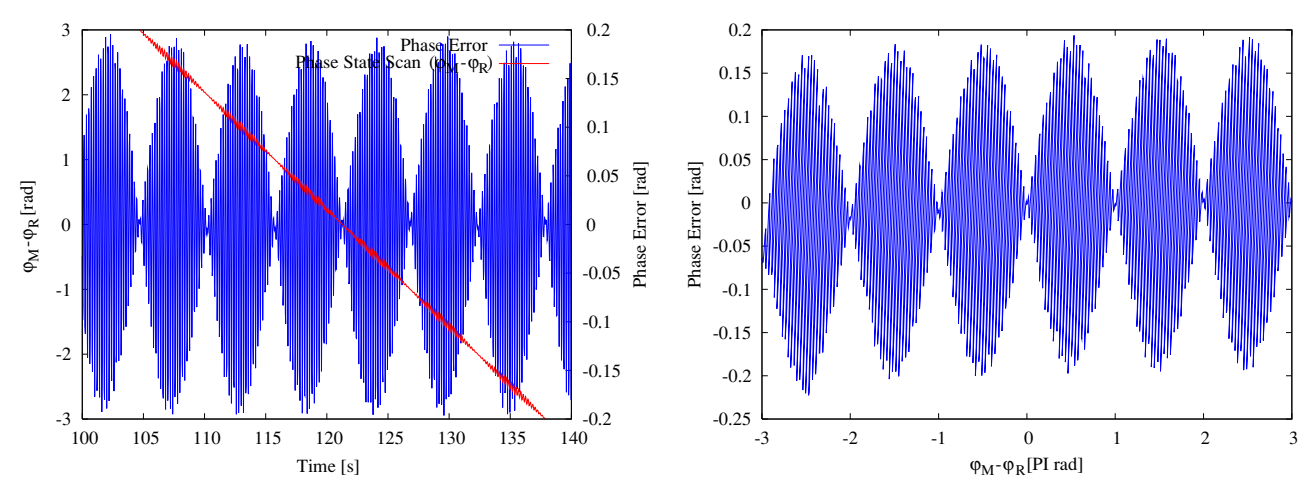

Figure 6. Phase $\varphi=\varphi_{\mathrm{M}}-\varphi_{\mathrm{R}}$ and its error (linear drift substracted) when a strong AM at an offset $2 \times f_{\text {het }}$ is added to the RF signal. This illustrates and confirms the $\sin \left(\varphi_{M}-\varphi_{R}\right)$ term in equation 9 .

- The spectrum directly at the output of the temperature compensated voltage controlled oscillator (TCVCXO) is free of any relevant sidebands that could disturb the phase measurement.

- The RF signal is disturbed in the RF-amplifier by electro-magnetic pickup of the amplified RF-signal of the second AOM driver. This generates a single sideband (SSB) in the spectrum that is spaced by $f_{\text {het }}$ from the local carrier.

- Due to the nonlinear amplification process in the RF amplifier, this SSB causes multiple phase modulations of the $80 \mathrm{MHz}$ carrier at the modulation frequencies $f_{\mathrm{m}}=\{1,2, \ldots\} \times f_{\text {het }}$ with decreasing power for higher sideband orders. (For the described phase error only the first two orders are relevant.)

\section{Noise mitigation}

As shown in the formulae (8) and (9) in section 4 the residual phase error $\delta \varphi$ depends on the sum and the difference of the phases $\varphi_{\mathrm{M}}$ and $\varphi_{\mathrm{R}}$. These two phases are dominated by environmental noise acting on the unstable part of the interferometers including the optical fibres. The main idea to mitigate the resulting noise is to keep the reference phase $\varphi_{\mathrm{R}}$ constant which also removes the environmental noise from the measurement phase $\varphi_{\mathrm{M}}$. Therefore an optical pathlength difference (OPD) stabilization has been implemented in the prototype modulation bench. A phase-locked loop (PLL) locks the optical reference phase $\varphi_{\mathrm{R}}$ to the electrical reference phase $\varphi_{\mathrm{el}}$ by compensating fluctuations of the optical pathlength difference $\Delta_{\mathrm{F}}$ (see figure 4). The bandwidth of the PLL is approximately $10 \mathrm{~Hz}$. Only with this OPD stabilization have the noise level requirements for the phase measurement been achieved. For the flight model two different OPD actuators were investigated: a free beam PZT actuator and a ring PZT with an optical fibre coiled around it. Both actuators showed the sufficient performance to reach the required sensitivity of the OMS.

\section{Performance measurements}

Current performance measurements of the longitudinal phase noise of the interferometer are shown in figure 7 . The goal of about $10 \mathrm{pm} \mathrm{Hz}^{-\frac{1}{2}}$ for the longitudinal phase sensitivity has been achieved in the relevant frequency range from $3 \mathrm{mHz}-30 \mathrm{mHz}$. The angular resolution 


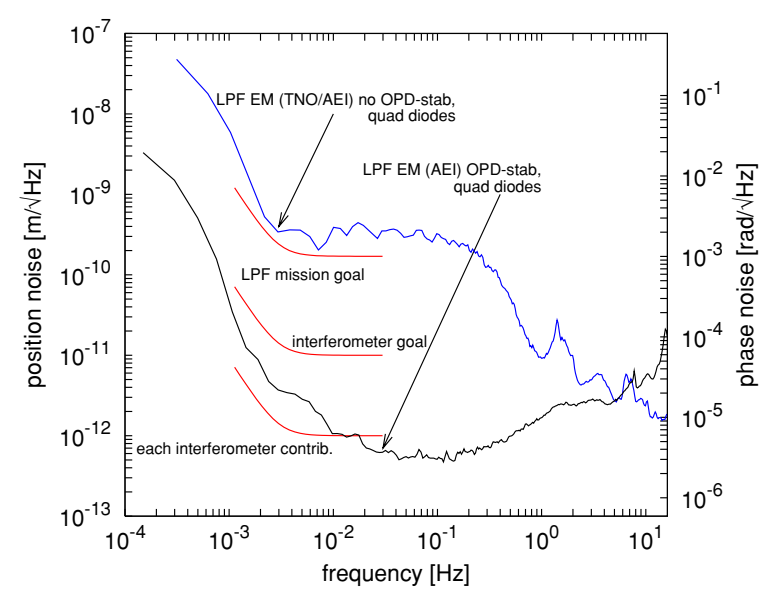

Figure 7. Noise curves of the phase measurement. The upper curve shows the sensitivity when the differential phase is not being stabilized. The lower curve shows the current sensitivity with the OPD- and laser-power stabilization (see section 4).

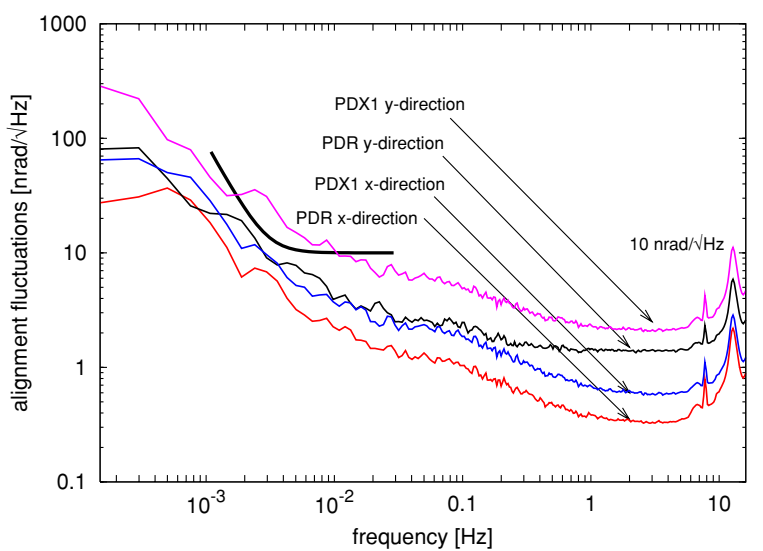

Figure 8. This graph shows the sensitivity of the alignment measurements of the reference interferometer (PDR) and the interferometer that measures the alignment of test mass 1 (PDX1).

of the interferometer for sensing test mass tilt and rotation perpendicular to the sensitive $x$-axis (see figure 8) reaches an accuracy better than $10 \mathrm{nrad} \mathrm{Hz}^{-\frac{1}{2}}[1]$.

\section{Inhomogeneous phase fronts}

Phase noise measurements showed a higher noise level by using quadrant photo diodes (QPD) in comparison with results obtained with single element diodes (SED). A possible explanation is given by the combination of beam jitter over the quadrants with an inhomogeneous spatial distribution of the phase caused by differences between the parameters of the interfering beams. Some information may be overlooked at the slit separating the quadrants which is typically $50 \mu \mathrm{m}$ wide. In order to analyse and match the wavefronts, a spatially resolving phase measurement system has been developed $[4,5]$. This instrument is very helpful for an 


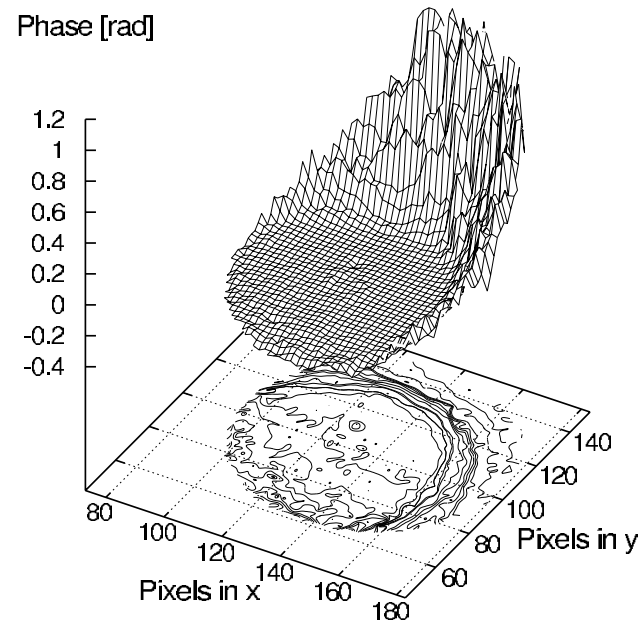

Figure 9. Spatial distribution of the phase measured at the frequency interferometer of the LPF optical bench $[4,5]$.

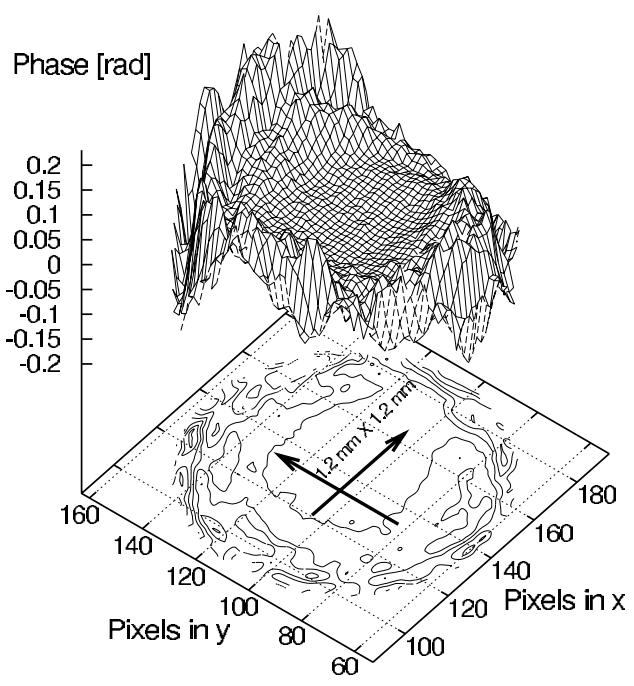

Figure 10. Homogenized phase front: spatial distribution of an optimized phase measured at a tabletop interferometer $[4,5]$.

optimal adjustment of an interferometer and will therefore be used in the production of the flight model of the optical bench for LTP (see figures 9 and 10).

\section{Conclusion}

We have demonstrated the required performance of $10 \mathrm{pm} \mathrm{Hz}^{-\frac{1}{2}}$ for the LTP optical metrology. Our investigation of the residual noise sources that spoil the performance of the phase measurement identified spurious sidebands spaced from the carrier by the heterodyne frequency and its harmonics as the origin of the main noise source. We have found an analytic 
description of the phase error induced by these sidebands and have demonstrated a method to mitigate this effect.

\section{References}

[1] Heinzel G et al 2004 Class. Quantum Grav. 21 581-7

[2] Heinzel G et al 2006 J. Phys.: Conf. Ser. 32132

[3] Elliffe E J et al 2005 Class. Quantum Grav. 22 257-67

[4] Guzmán F 2004 Master's Thesis AEI Hanover and University of Oldenburg

[5] Guzmán F et al 2006 in preparation

[6] Robertson D et al 2005 Class. Quantum Grav. 22 155-63

[7] García A F et al 2006 Class. Quantum Grav. 23 S133 